SECTIOR VI

BNVIRONMENTAL ASPECTS OF NUCLEAR ACTIVITY 


\title{
RELATIONSHIPS BETWEEN SEYFERT GALAXIES, QSOs, AND BL LACs
}

\author{
J.B.Hutchings \\ Dominion Astrophysical Observatory \\ 5071 W.Saanich Rd, Victoria, B.C., Canada
}

\section{INTRODUCTION}

In this review, I will deal principally with the optical morphology of these three types of object, and attempt to outline similarities and essential differences. By this, I mean to include studies of the types of host galaxy, nuclear and galaxy luminosities and colours, interactions with other galaxies, and the general associations with other galaxies. Where relevant, I will refer to morphology at other wavelengths (generally radio), spectroscopy, and UV or X-ray properties. However, I expect these areas to be covered much more fully by other reviews and papers in this volume.

The first point to deal with is the general distinction between QSOs and Active Galaxies in general. While few people now doubt that QSOs are active galaxy nuclei, there still remains much (to my mind) pointless distinction between 'real' QSOs and mere AGN. I hope to help dispell the distinction, but for general classificatory purposes I will follow the $\mathrm{M}_{V}-21.5$ (for $\mathrm{H}_{0}=100$ ) demarkation. However, since magnitudes usually quoted are for the whole system, and nuclear to galaxy luminosity ratios vary from 0.1 to 10 (at least), even this 'definition' is hard to enforce, and I do not take it very literally in what follows.

QSOs

In recent years, there have been several major papers on the morphology of the host galaxies of QSOs (the most comprehensive being Gehren et al 1984, Malkan 1984, Malkan et al 1984, Hutchings et al 1984a,b, Stockton 1986, and Smith et al 1986). These, and many other investigations of smaller samples all agree that low redshift $(<0.5)$ QSOs are embedded in faint nebulosity that resembles in every way fairly luminous galaxies at the QSO redshift. Spectroscopic investigations of a small sample of these bear out this conclusion by finding absorption line systems or extended emission line systems at the same redshift as the QSO nucleus.

The information that can be extracted from imaging data depends quite strongly on the spatial resolution and faint limit achieved. Nevertheless, there are several points of strong agreement between independent investigators, with very different types and quality of data. These are that the host galaxies are in general luminous, but do have a considerable range of luminosity, that it is very difficult to determine their types, and indeed that a very large fraction of them have peculiar morphology, or are currently interacting tidally with another galaxy. It is frequently supposed that such interactions 
are a common or even necessary way of activating the nuclear activity. However, there have been overoptimistic claims as to the galaxy type on the basis of fitting models to measured luminosity-radius curves, and data with lower spatial resolution have often missed spotting nearby companions or morphological peculiarities.
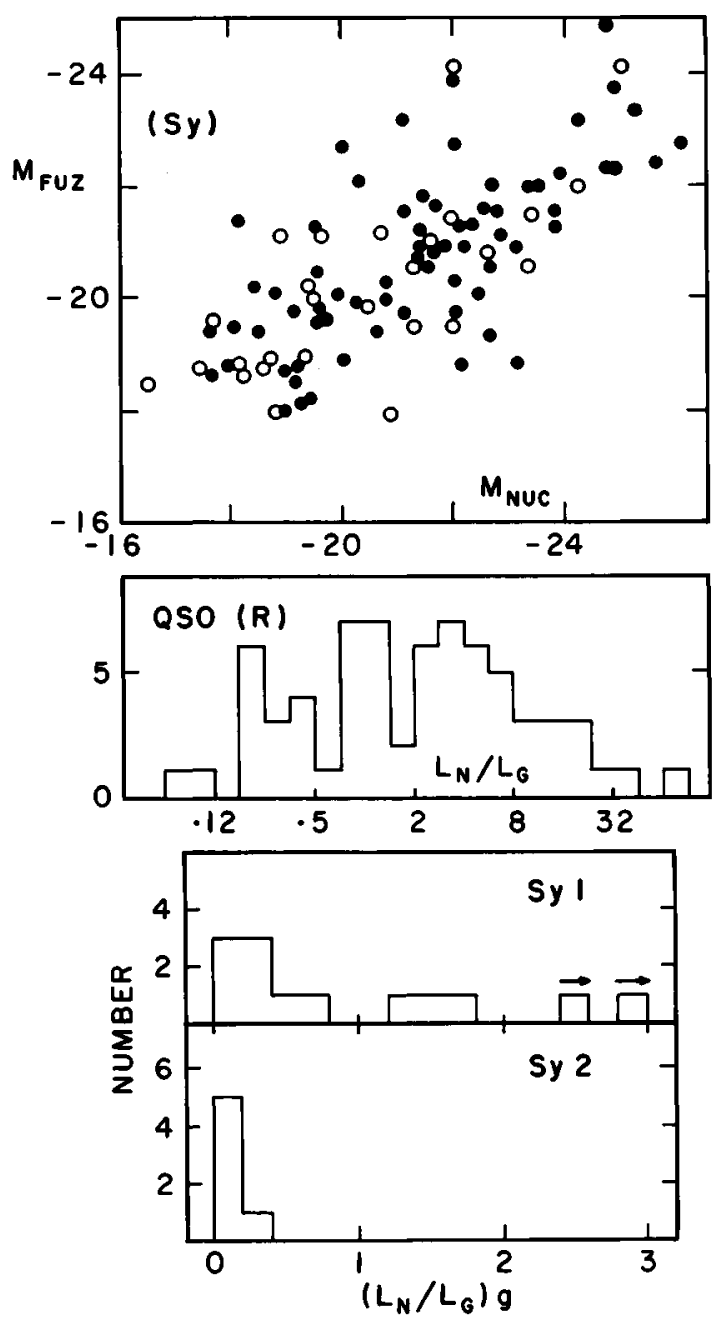

Figure 1. Nuclear and galaxy luminosities of QSOs and Seyferts. Upper: Resolved objects in HCC. Open symbols are B, filled are R. (Sy) indicates region populated by Seyferts. Lower right region is empty. Centre: Distribution of ratio of Nuclear to Galaxy luminosity from HCC sample, in R light. Lower: distribution of ratio of Nuclear to Galaxy light for Seyferts by Yee (1983). Note overlap of QSO and Sy distributions. 


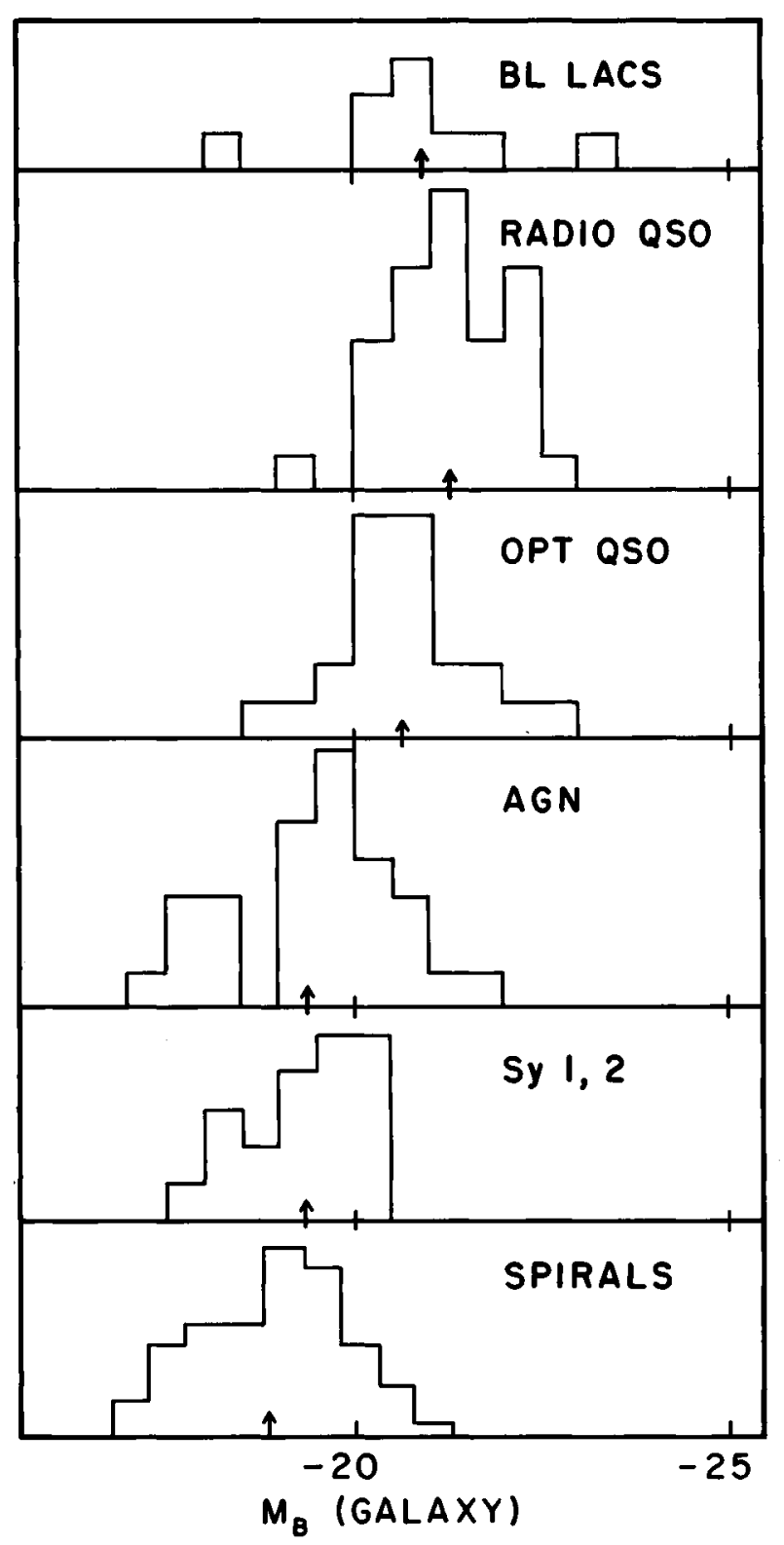

Figure 2. Distributions of $B$ absolute magnitudes of host galaxies, from the literature cited, for $\mathrm{H}_{0}=100$. AGN and QSOs are separated at (Galaxy + Nucleus) -21.5. Arrows indicate mean values. Note progression to brighter host galaxies from bottom to top of diagram. 
Hutchings et al (1984) illustrate some important distributions of QSO nuclear and host galaxy properties (see Fig 1). There is a wide range of luminosities and of nuclear to host luminosity ratios. There is, however, an apparent correlation between the two, in the sense that more luminous galaxies host more luminous QSOs. This diagram (Fig 1:upper) must be further populated in the upper right by Sy galaxies, but the absence of unresolved objects means that there is an upper limit to nuclear luminosity for a given galaxy luminosity. Since most of the galaxies are quite luminous, and since high luminosity galaxies are rare, it also follows that with increasing galaxy luminosity the chance of having a QSO nucleus increases, and is close to unity for galaxies of $\mathbf{M V}_{V}-23$ or brighter.

The distribution of nuclear to host luminosity ratios is broad, and overlaps that of Seyfert galaxies. Also, the distribution of isophote axis ratios resembles that of Seyferts, and not that of either normal spirals or normal ellipticals. It is also notable that a number of isophotes show a rotation of $90^{\circ}$ as they go fainter, which is characteristic of barred galaxies. In the sample of Hutchings et al, some $15 \%$ of the objects do this. Further, several investigations have noted that structure (arms, blobs, tails, etc) is seen in blue light, again characteristic of spiral galaxies or those with active star formation. On the other hand, few, if any, signatures of purely elliptical galaxies have been found.

The radio-loud quasars in general reside in more luminous galaxies with larger scale lengths than the radio-quiet QSOs. This result probably contains some selection effects: radio-loud quasars are rarer and thus tend to have higher redshift than optically selected objects - this leads to systematic differences in the ease of resolving the host. In addition, radio-loud objects are also optically loud, again leading to a bias. Both Gower and Hutchings (1984) and Machalski and Condon (1986) find a correlation between radio core and optical nuclear luminosity, so that the two appear to be related in any case. Finally, there is evidence that there are two types of radio luminosity: the classical radio quasars, and a distribution of much lower radio fluxes from almost all QSOs, which suggest that there may be a beamed flux in all objects, whose apparent luminosity depends on the orientation of the central source to our line of sight. There is even some evidence (Hutchings and Gower 1985) that some of the optical flux is beamed. Overall, however, there still remains fairly convincing evidence that the very luminous radio quasars reside in more luminous galaxies. Figure 2 shows the distributions of various representative samples of QSO host galaxies, along with other objects we discuss further below.

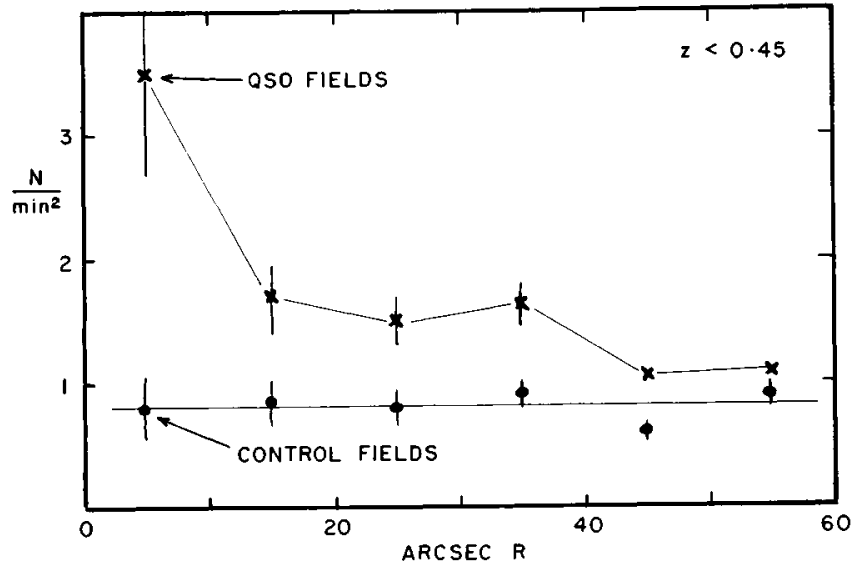

Figure 3. Counts of galaxies within radius $R$ of $Q S O s$ and control objects, from Yee and Green (1984), showing strong clustering very near to QSOs. 
A second major investigation of QSO environments is that of nearby galaxies. The most thorough work on QSOs was done by Yee and Green (1984). Their results are restricted by the faint limit of their data, but show that for redshifts 0.4 or less, QSOs live in very rich but compact groups of galaxies (Fig 3). There is some indication that steep spectrum (large) radio quasars are in richer environments. Further, Hintzen et al (1983) and Hintzen (1984) find that C-shaped, or tailed sources appear to be in clusters of galaxies. Motion through the IGM of such clusters is the likely cause of the swept-back radio structure. Optical spectroscopic work on companions to QSOs by Stockton (1978) and Heckman et al (1984) has shown that these galaxies are essentially all at the same redshift as the QSOs. More recent work by Yee and Green, and others, has concentrated on the redshift range 0.5 to 1.0 with deeper imaging: these show that a high fraction of these objects reside in very rich clusters. Thus, the environment has evolved. De Robertis (1985) suggests that this is consistent with the activation of all QSOs by interaction and gas transfer: as cluster environments evolve, the sites suitable for QSO activation move from rich clusters to small groups, where virialisation takes longer. This in turn leads to QSO density evolution consistent with number-redshift counts.

Stockton (1986) has obtained narrow-band images of a large sample of QSOs in the light of $[\mathrm{O} \mathrm{III]}]$. These reveal the presence of large irregular clouds of ionised gas around many QSOs with strong narrow-line regions, whose morphology is generally unrelated to the continuum light. These results indicate that there is extensive and disturbed gas, highly suggestive of tidal interactions, which is photoionised by the nuclear radiation. Velocities in some cases are large with respect to the QSO nucleus (e.g. MacKenty and Stockton 1984, Hickson and Hutchings 1986). The full interpretation of these discoveries will need more detailed spectroscopy.

The interactions seen in many (30-40\%) QSOs are generally with a smaller galaxy (although there are a few cases where twin tidal tails indicate comparable masses). Gaskell (1985) has proposed that QSOs are activated by mergers of luminous galaxies and dwarf companions, noting that most local large galaxies have such companions. He further notes that such mergers would lead to the formation of binary compact nuclei, which in turn offers an explanation for the asymmetry of broad emission lines and their different velocities from the narrow lines.

Finally, a word on off-nuclear spectroscopy. This is difficult and time-consuming, and results are accordingly noisy and sparse. Oke and Boroson and collaborators (e.g. Boroson et al 1985) have suggested that there are two types of QSO 'fuzz': blue with emission lines and red without. These two types seem to correspond to extended, steep spectrum radio sources, and compact flat spectrum radio sources. The dichotomy may be explained in terms of accretion rates through a disk, which becomes thicker and cooler and less able to collimate radio jets as the accretion rate increases. Other results from offnuclear spectroscopy include the confirmation of absorption redshifts close to those of the QSO nucleus, the occasional presence of a young stellar population, and the measurement of the ionised gas velocities.

The gas in QSO galaxies may also be studied by its $\mathrm{H} \mathrm{I} 21 \mathrm{~cm}$ line. There are only a few 'real QSO' detections here due to redshift and sensitivity limitations, but the indications are (fig 4) that $\mathrm{H} \mathrm{I}$ is present, with unusually broad profiles (tidal disturbance?), somewhat weak (ionised?), and differing in velocity from the optical narrow lines by $100-200 \mathrm{~km} . \mathrm{s}^{-1}$ (again tidal effects?). As we see below, there are similar results for Seyferts. 

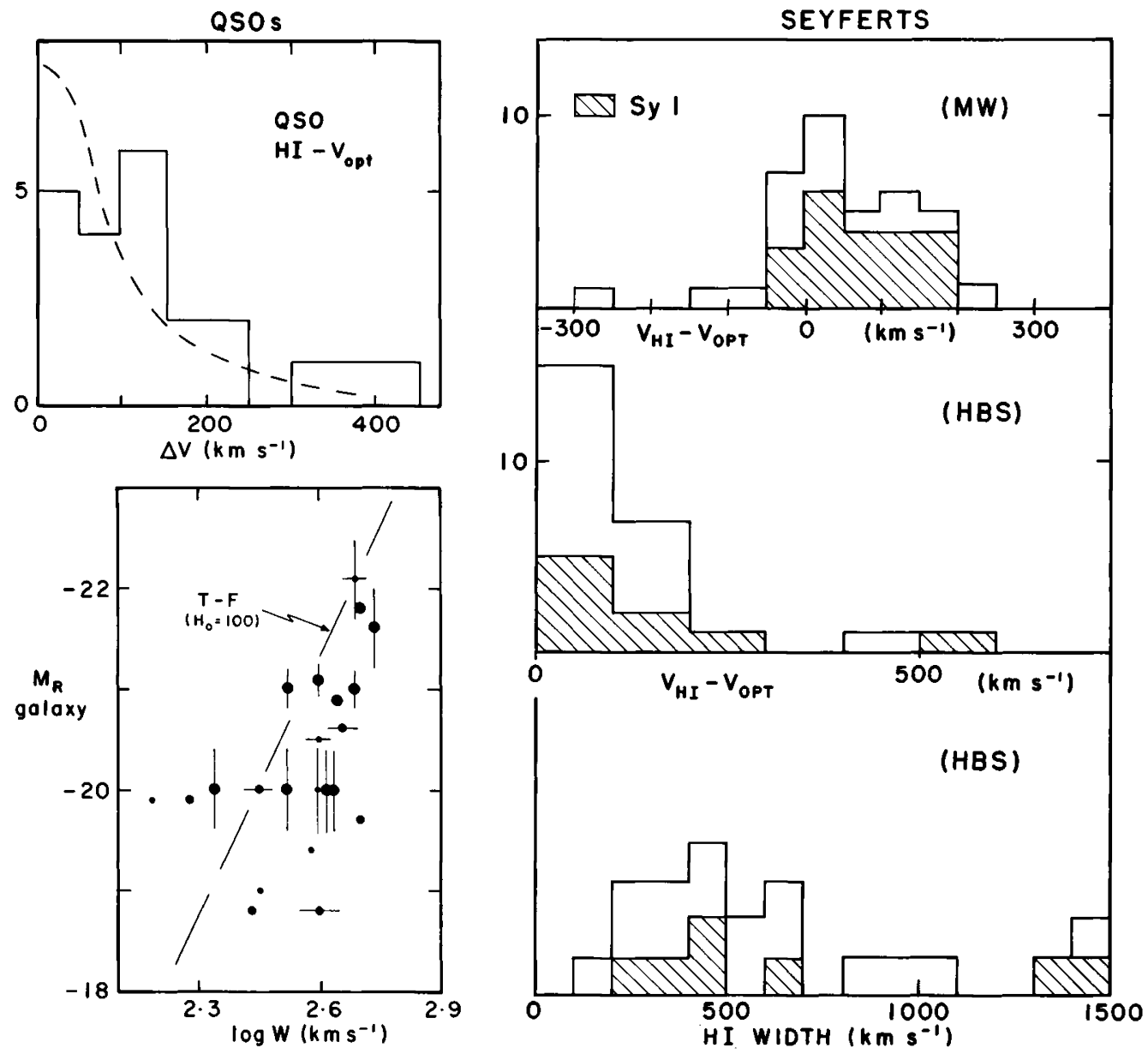

Figure 4. H I $21 \mathrm{~cm}$ measurements of QSOs and Seyferts. Left top: Misalignment of H I and [O III], with expected distribution. Left bottom: Line width vs $\mathrm{M}_{\text {gal }}$, and T-F line, showing general excess line width. Right upper two: H I - [O III] misalignments for Seyferts from two cited papers. Right bottom: H I widths, showing a few very large values. 


\section{SEYFERT GALAXIES}

The morphology of Seyfert galaxies is easier to study as they are more common (hence much closer) and have less bright nuclei, than QSOs. It is well known that Seyfert nuclei are found only in spiral galaxies. The counterparts among ellipticals are the broadlined radio galaxies, which will not be dealt with in this paper. Classic studies of Seyfert galaxies are by Adams (1977), and Simkin, Su, and Schwarz (1980). These investigations note that many Seyferts have peculiar morphology or are barred galaxies, compared with non-active galaxies. Yee (1983) studied the galaxy morphology and luminosity of a sample of Seyferts (see figures 1,2). His result was that Seyfert galaxies are luminous, but less so than QSO host galaxies, on average. The nuclei of Seyfert 1 galaxies are bluer than Seyfert 2, and the ratio of nuclear to galaxy luminosity is larger and ranges more widely in Sy 1 than in Sy 2 galaxies. Seyfert 2 galaxies are reported by Heckman et al (1978) to have no spiral arms, but later work does not seem indicate a significant difference from Seyfert 1's.

Lawrence and Elvis (1982) and Fabbiano et al (1984) have suggested that Sy 2 and NLRGs are simply Sy 1 and BLRGs with heavy obscuration of the nucleus. Examples of each have been claimed (Antonucci and Miller 1985 and Fabbiano et al 1986). However, it seems to me that while this obviously can explain some cases, it may not account for all the morphological differences between them. Also, there is at least one counterexample in Mkn 231, a Sy 1 galaxy with very heavy ( $A_{V} \sim 2$ mag) nuclear reddening. The issue here is perhaps not one of reddening but one of obscuration of the nucleus by its accretion disk, which in general is not aligned with the galactic plane. The observation of broad line wings in polarised (scattered) light (Miller: this symposium), is powerful evidence for this idea in NGC 1068, for example.

An important investigation into the environment of Seyfert galaxies was done by Dahari (1984). The closest companion galaxies of 93 Seyfert galaxies were investigated, together with a large number of control galaxies. After making allowance for chance superposition of galaxies in the line of sight, and carefully defining the criteria for companionship, he finds that $15 \%$ of Seyferts have genuine close companion galaxies, compared with $3 \%$ of the control galaxies. $7.5 \%$ have large companions, compared with $1 \%$ for control objects. A later investigation showed that the companions to Seyferts do lie at the same redshift. Thus there is a clear association of tidal effects and Seyfert activity. In a separate investigation of 167 interacting galaxies, Dahari (1985) finds only a small excess of Seyferts over the $1 \%$ 'universal average'. In extreme interaction systems, he finds no Seyferts. Bushouse (1985) in a similar investigation finds that galaxy interactions do not invariably lead to star-formation. Fricke (1986) finds evidence for 'clustering' of active galaxies within $200 \mathrm{Kpc}$ of Seyferts, and suggests that these galaxies may be in a state of activation triggered by interactions a considerable time earlier. Thus, tidal effects may be neccesary but not sufficient to cause nuclear (or other) activity in galaxies. If, as generally supposed, the process is accretion by a central massive body, the other necessary conditions may be the presence of gas in the tidal interaction, angular momentum configurations suitable to bring it in to the nucleus, and the presence of the central object. Finally, the relative lifetimes and time-lags between interactions and nuclear activity are unknown, but important in this scenario.

There is a lack of edge-on Seyfert galaxies, which is probably due to disk reddening obscuring the nucleus in such objects. Cheng et al (1983) show that there is a colour dependence of the disk inclination in known Seyferts which confirms this. The similar lack of edge-on QSO galaxies may be an indication of the same selection effects, and the reason that the axial ratio distributions for the two groups are similar. Note, however, that the only resolved BAL QSO - 1411+442 - is not edge-on, although it is moderately 
flattened. Its nuclear colour is not unusually red. On the other hand, the reddened Seyfert Mkn 231 is a merger, but not significantly flattened. Thus, as discussed above, the presence of absorbing and reddening material is not necessarily confined to a disk plane.

Seyfert galaxies are found to be weak and compact radio sources. The weakness is probably due to stopping of radio emitting plasma by gas in the systems, as is generally supposed for radio-quiet QSOs (see review by Wilson in this volume). Meurs and Wilson (1984) discuss radio (and optical) luminosity functions for Seyferts, and note that the Sy 2 galaxies are more luminous than Sy 1 . Here too, the stopping power of the (BLR) gas is thought to suppress the Sy 1 radio fluxes. Studies of the $H I 21 \mathrm{~cm}$ line are more extensive than for QSOs, but the results are similar (Heckman et al 1978, Mirabel and Wilson 1984: see Fig 4). H I is generally present, but anomalous compared with normal spirals in more than $1 / 3$ of objects. The anomalies are generally broadness of profile, low (or high) H I flux, and the presence of absorption. Anomalies are more marked in objects with higher luminosity nuclei. Systematic differences between NL and H I velocities of order $60 \mathrm{~km} . \mathrm{s}^{-1}$ are noted by Mirabel and Wilson.

It would be of interest to explore the similarities between the Seyferts and QSOs in terms of the presence of extended emission line regions. In spectroscopic data I have on low luminosity QSOs, the phenomenon seems to be common, but there does not appear to be a systematic study of normal Seyferts. However, Mackenty (1986), and Hutchings and Neff (1986) report finding jet-like features in [O III] light in Mkn 315 and Mkn 231 respectively.

Finally, we should mention a class of Seyferts which have double or multiple nuclei. Petrosian et al (1978) have picked out a number of these, and there are studies of some of them. Often, only one nucleus is a true Seyfert, while the other may be more like an H II region or LINER. Relative velocities are of the order of a few hundred km.s ${ }^{-1}$. It appears that these objects are in a short-lived state, probably of merging, as they have many characteristics of merging or colliding galaxies (e.g.Mkn 110, Mkn 266, Mkn 231, Mkn 463, 3C 75). The presence of two centres of activity may be a manifestation of the Gaskell scenario, or it may be that a short-lived period of star-formation is occurring as a result of the merging process.

Overall, Seyfert galaxies have a continuity of galaxy properties with QSOs, as well as of nuclear spectra and luminosities. These properties overlap so deeply that it makes sense only to assume that they are essentially the same phenomenon.

\section{BL LAC OBJECTS}

The third category of objects to be covered here, the BL Lacs, or Blazars, are more enigmatic. They have many extreme properties, such as rapid variability, core-dominated radio fluxes, high polarisation, while also having very featureless spectra. Many of these properties can be explained in a qualitative way by the assumption that most of the observed flux is relativistically beamed towards us. Most of the work done on these objects deals with multiwavelength monitoring - from radio to $X$-ray - and variability studies, which do not fall within the scope of this paper. I will try to cover the morphological studies which may relate the BL Lacs with the QSOs and Seyferts already mentioned.

There are not yet many very extensive or convincing investigations along such lines. There is a widely quoted belief that BL Lacs are nuclei of giant Elliptical galaxies, but a close investigation of the literature reveals that while this may be reasonable, the belief is based on consistency arguments rather than really indisputable evidence. Such arguments are given by Weistrop (1983) in showing that the spectrum flux distribution is similar to a giant elliptical in the case of $1727+50$, or that the absolute magnitudes are about right for 
a few objects. In others, the luminosity profiles are claimed to be inconsistent with a spiral $(1219+28$ in Weistrop et al 1985). Ulrich et al (1975) and Mufson and Hutter (1981a,b) quote similar consistency arguments for several objects. Hutchings et al (1984) studied three BL Lac objects and were unable to determine a galaxy type from the luminosity profiles. I have an excellent CCD image of BL Lac itself, and even that does not yield a clean $r^{1 / 4}$ law, probably because of the presence of some faint companions or superposed objects (Fig 5). Nevertheless, it is difficult to make a definitive test for an elliptical, and in spite of the uncertainty, I am inclined to believe the general prejudice in this regard. Figure 2 shows that the few measured host galaxy luminosities do have a high median value.
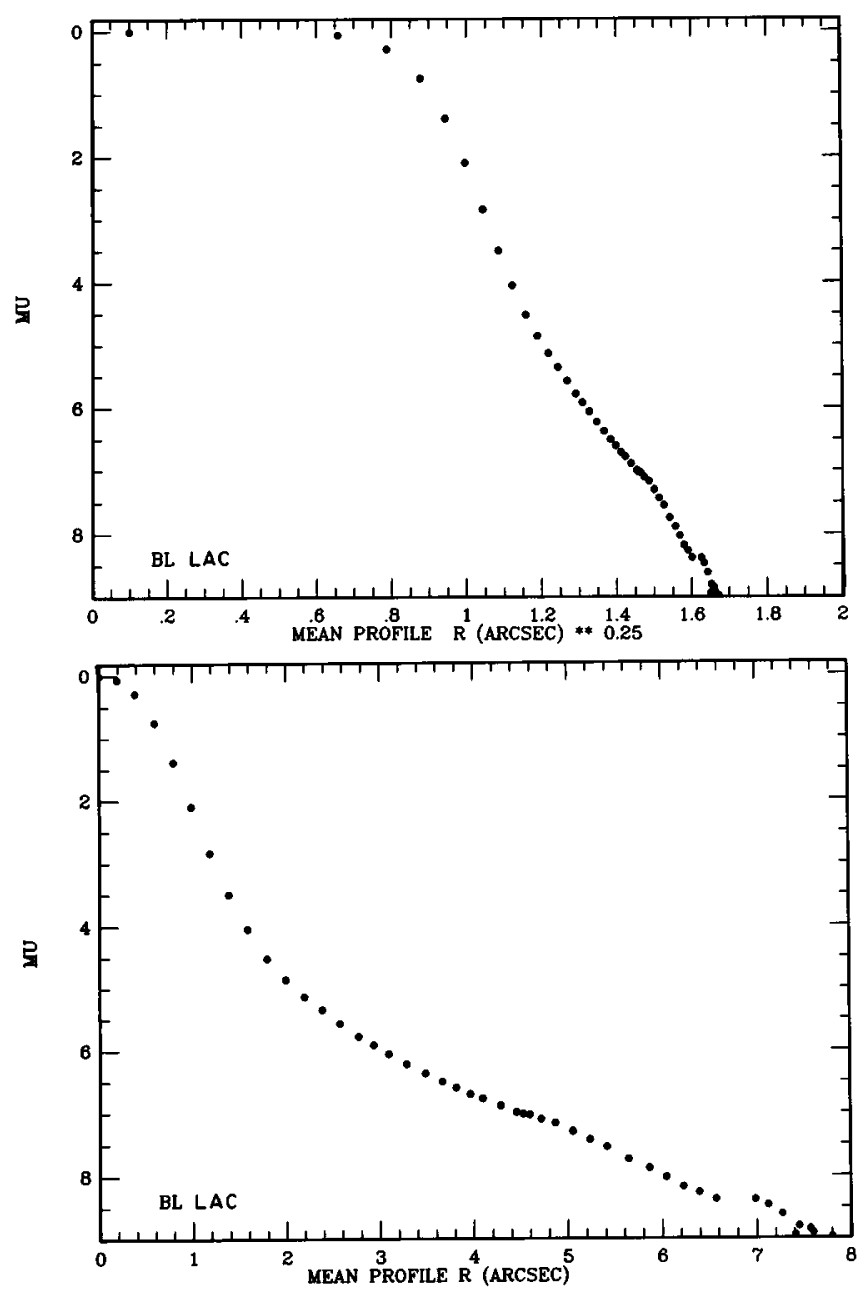

Figure 5. Luminosity profile of BL Lac from B band CCD image. Note that the plot is not linear on either $\mathrm{r}^{1 / 4}$ or exponential law, indicating that the host galaxy is non-standard. 
The redshifts of BL Lac objects are often unknown because of the lack of emission lines in the nuclear spectra. However, many of them have resolved host galaxies, and some of those have absorption line redshifts. Redshifts of such objects are generally low ( 0.2 or less), and consequently it is generally supposed that BL Lac objects are a lowredshift population, with a higher local space density, lower luminosity, and different cosmic evolution from normal QSOs. X-ray data have also been incorporated into such scenarios (e.g Maccacaro et al 1984). This brings us to an important cautionary point. The apparent low redshift of BL Lacs is certainly to some extent a selection effect, and it is necessary to obtain redshifts of a representative sample before proceeding too far with cosmic models. To this end Antonucci and Hickson (private communication) are obtaining high $\mathrm{S} / \mathrm{N}$ spectra to try to detect faint emission lines for redshift determination. Results so far are sparse, but the first redshift they have is $\mathbf{0 . 6}$, already very high for a BL Lac.

There has been important radio morphological work on BL Lacs in recent years, which should at least be mentioned here. Most, if not all, of these objects have extended structure, (which is small and complex) as well as a very strong unresolved nuclear source, and it has been argued (Wardle et al 1984, Antonucci and Ulvestad 1985) that these structures are end-on double-lobe sources, thus consistent with the beaming model for BL Lac nuclei. The extended structure has a typical luminosity similar to FR type 1 sources, according to Wardle et al.

I will not attempt more than a superficial summary of the models for BL Lac objects here. If the simple beaming model is right then it is necessary to know the amount of relativistic doppler boosting in intensity, and the beam angle, to know the true luminosity of the objects, and their true space density. Orr and Browne (1982) have proposed a 'unified model' to explain many radio sources by beaming, and again there is not space here to do it justice. Questions which arise are: can we recognize sideways-on objects of this kind? Are they normal radio galaxies? Are the nuclear emission lines truly suppressed (by high accretion rates and softening or shadowing of the nuclear radiation), or are they simply hidden by the boosted continuum flux? Statistics are ambivalent at present. I feel that detailed imaging studies and off-nuclear spectroscopy may help with this point. Certainly, less work has been done on these objects than the other active galaxies, so far. In this respect, the statistics of normal radio galaxies, and the interaction scenario for both them and BL Lacs are relatively unexplored, although programs are presently under way. From these it is already clear that many radio galaxies which are not quasars, are interacting. Thus, we need to look for some of the other parameters involved in activating nuclei. They may be detected by imaging work, or they may be more subtle, like the nuclear accretion and spin rate scenario of Blandford.

\section{CONCLUSION}

While morphological studies have provided us with many vital clues to the environments of QSOs and AGN, they have raised further questions, and such studies need to be continued on statistically meaningful samples, with particular emphasis on redshift, optical luminosity, and radio morphology as important parameters. I feel that we are close to a unified understanding of active galaxies in terms of accretion by central massive objects, with principal parameters of central mass, angular momentum, accretion rate, and the details of the central gas density and flow. Stockton (1986) has summarised many of the probable ways in which these things vary in QSOs, and it seems likely that other active galaxies represent other areas in these parameter spaces. Future work will need to address the relationships between Seyferts, radio galaxies, and BL Lacs with QSOs. As well as imaging, spatially resolved spectroscopy is very important. While HST will doubtless make important studies on a few key objects, this work needs to be done primarily with large amounts of time and collecting area, and hence from the ground. 


\section{REFERENCES}

Antonucci R.R.J., Ulvestad J.S., 1985 Ap.J. 294, 158.

Antonucci R.J., Miller J.S. 1985 Ap.J. 297, 621.

Adams T.F. 1977 Ap.J. Suppl. 33, , 19.

Boroson T.A., Persson S.E., Oke J.B. 1985 Ap.J. 293, 120.

Bushouse H.A. 1986 A.J. 91, 255.

Cheng F.Z., Danese L., de Zotti G. 1983 M.N.R.A.S. 204, 13P.

Dahari O. 1984 A.J. 89, 966.

Dahari O. 1985 Ap.J. Suppl 57, 643.

De Robertis M.M. 1985 A.J. 90, 998.

Fabbiano G. et al 1984 Ap.J. 277, 115.

Fabbiano G. et al 1986 Ap.J. 304, L37.

French H., Gunn J.E. 1983 Ap.J. 269, 29.

Fricke K.J., Kollatschny W., Loose H.H. 1986 Structure \& Evol. of AGN: Reidel p551.

Gaskell C.M. 1985 Nature 315, 386.

Gehren T, Fried J., Wehinger P.A., Wyckoff S., 1984 Ap.J. $278,11$.

Gower A.C., Hutchings J.B. 1984 A.J. 89, 1658.

Heckman T.M., Balick B., Sullivan W.T. 1978 A p.J. $224,745$.

Heckman T.M., Miley G.K., Green R.F. 1984 Ap.J. 281, 525.

Hickson P., Hutchings J.B. 1986 Ap.J. (preprint)

Hintzen P., Ulvestad J., Owen F.N. 1983 A.J. 88, 709.

Hintzen P. 1984 Ap.J. Suppl 55, 533.

Hutchings J.B., Crampton D., Campbell B., 1984 Ap.J. 280, 41.

Hutchings J.B. et al 1984 Ap.J. Suppl 55, 319.

Hutchings J.B., Gower A.C. 1985 A.J. 90, 405.

Hutchings J.B., Neff S.G. 1986 (preprint)

Hutter D.J., Mufson S.L. 1981 A.J. 86, 1585.

Lawrence A., Elvis M. 1982 Ap.J. 256, 410.

Maccacaro T. et al 1984, Ap.J. 284, L23.

Machalski J., Condon J.J. 1986 A.J. 91, 998.

MacKenty J.W. 1986 (preprint)

MacKenty J.W., Stockton A. 1984 Ap.J. 283, 64.

Malkan M. 1984 Ap.J. 287, 555.

Malkan M., Margon B., Chanan G.A. 1984 Ap.J. 280, 66.

Meurs E.J.A., Wilson A.S. 1984 A. and Ap. 136, 206.

Mirabel I.F. and Wilson A.S., $1984 A$ p.J. $277,92$.

Mufson S.L., Hutter D. 1981 Ap.J. 248, L61.

Orr M.J.L., Browne I.W.A. 1982 M.N.R.A.S. 200, 1067.

Petrosian A.R., Saakian K.A., Khachikian E.E. 1978 Astrofizika 14, 69.

Simkin S.M., Su H.J., Schwarz M.P. 1980 Ap.J. 237, 404.

Smith E.P. et al 1986 Ap.J. (preprint)

Stockton A., MacKenty J.W. 1983 Nature 305, 678.

Stockton A. 1978 Ap.J. 223, 747.

Stockton A. 1986 Ap. and Sp. Science 118, 487.

Ulrich M-H et al 1975 Ap.J. 198, 261.

Wardle J.F.C., Moore R.L., Angel J.R.P. 1984 Ap.J. 279, 93.

Weistrop D 1983 IAU Symp 97: Reidel Co p377.

Weistrop D. et al 1985 Ap.J. 292, 614.

Yee H.K.C. 1983 Ap.J. 272, 473.

Yee H.K.C., Green R.F. 1984 Ap.J. 280, 79. 


\section{DISCUSSION}

GURVITS: 1 . Are known any sources with radio jets and fuzz?

2. If there are some sources is there any correlation between 'MNUC MFUZ' ratio and 'Fjet/Fcore' ratio, especially for VLBI-jeis?

HUTCHINGS: 1) Yes, but the radio and optical structures are generally uncorrelated. There is a tendency for optical and radio axes to be aligned or orthogonal (Gower \& Hutchings, A.J. 1984).

2) There is no such correlation. In radio-weak sources, there is some evidence for radio and optical beaning (Hutchings + Gower, A.J. 1985), comparing F core with MNUC/MFUZ.

TOVMASSIAN: Speaking aboui interacting galaxies you said that the radio emission from them and the other signs of activity are due to a tidal effect. Similarily the jets were explained by merging galaxies. I know that these are widely accepted ideas. But if it is really so, then how to explain the same type of events and features in the case oî definitely single galaxies.

HUTCHINGS: We notice that interactions are so comnon in active galaxies that we are forced to believe that the events are connected. There may be other ways as well to activate the nucleus as yet unknown. On the other hand, we may not be able to detect all interactions if they are close tn the bright nucleus or if there is a long delay between tidal encounter and subsequent nuclear activity. There are few objects, we can say are definitely single!

WILSON: Mirabel and I did an HI 21crin single dish survey of nearly 100 Seyferts a couple of years ago (Ap.J. 1983 or 1984) and Found that in general, the HI disks of Seyierts do not seem to be highly disturbed. Would you care to comment on your Seyferts with very wide $\left(1000 \mathrm{~km} \mathrm{~s}^{-1}\right) 21 \mathrm{~cm}$ lines?

HUTCHTNGS: The results I quoted are those published by Hecknan et al. fron earljer and smaller sample. Evidently your results supercede these and thus make the Seyferts more noriial than the QSOs. The large beam of the Arecibo dish may also include cormpanion objects and thus widen the profiles.

BARTEL: How did you distinguish between the nucleus and the fuzz for your calculation of $L$ (nucleus) and $L$ (fuzz)? Is such a distinction not subject to ratiner large uncertainties is not completely arbitrary?

HUTCHINGS: The procedure is described exactly in Hutchings et al. Ap.J. 1984. The numbers are corrected for $z$, beam size and dynamic range and are derived by subtracting the point spread function from the QSO inages.

ALLOIN: In the examples of multiple nuclei QSOs you showed, couldn't it be that, using narrow interierence filters, you pick up one nucleus in 
the continuuri and the other so-called nucleus in a sirong emission line, due to velocity differences between the two objects. In such a case you would have a single nucleus plus giant HII regions around.

HUTCHINGS: The examples I showed and referred to have all been imaged with one or more broad band Filter. There is no doubt there is continuun luminosity. However, not all nuclei have two Seyfert spectra, so the exact nature of the different nuclei is not always clear.

HECKMAN: Two cominents: 1) Bruce Balick and I have a (still unpublished) narrow-band imaging survey at a large sample of Seyferts. Our data indicate that large nebulae like those seen by Stockton around QSO's are rarely associated with Seyferts. 2) My collaborators and I have just conpleted a deep imaging survey of 43 radio galaxies at $z \sim 0.03-$ 0.20 which shows that a large fraction ( $1 / 3$ to $1 / 2)$ of powerful radio galaxies have strongly disturbed optical morphologies. This suggests to us that galaxy collision or mergers are important in the radio galaxy phenomenon (as in other types of AGN's/QSO's).

HUTCHINGS: The visibility of [OIII] clouds may well depend on the presence of a powerful source of radiation (i.e. the QSO rather than Sy nucleus).

MELNICK: In your last histogram spirals are $>2$ mag fainter than QSO galaxies. Does that mean that these are the results of multiple mergers?

HUTCHINGS: QSOs reside in lumirious galaxies, but in general the interactions we see are with much less luminous companions.

In addition, it is likely that QSO spirals (specially radio-loud ones) are more luminous than ellipticals. This is not a contradiction in terms of galaxy populations since, after all, QSO's are extremely rare. 